\title{
QCD transition temperature: approaching the continuum on the lattice
}

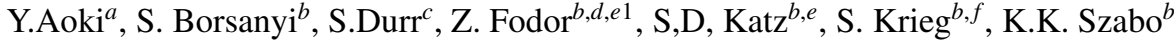 \\ ${ }^{a}$ RIKEN BNL Research Center, Brookhaven National Laboratory Upton, NY 11973 USA, \\ b Bergische Universität Wuppertal, Gaussstr. 20, D-42119 Wuppertal, Germany, \\ c NIC, DESY Zeuthen, D-15738 Zeuthen and FZ Jülich, D-52425 Jülich, Germany, \\ d Forschungszentrum Jülich, D-52425 Jülich, Germany, \\ e Institute for Theoretical Physics, Eötvös University, H-1117 Budapest, Hungary, \\ ${ }^{\mathrm{f}}$ Center for Theoretical Physics, Massachusetts Institute of Technology, Cambridge, Massachusetts 02139, USA.
}

\begin{abstract}
In order to clarify the source of the discrepancy between our previous transition temperature determination [Phys. Lett. B 643, 46 (2006)] and that of the Bielefeld-Brookhaven-ColumbiaRiken collaboration we improved our calculations by taking even smaller lattice spacings $\left(N_{t}=12\right.$ and $N_{t}=16$ at one point) and by using physical quark masses also for the $\mathrm{T}=0$ quantities. In addition to the kaon decay constant used for scale setting we determine four quantities (masses of the $\Omega$ baryon, $K^{*}(892)$ and $\phi(1020)$ mesons and the pion decay constant) which are found to agree with experiment. This implies that -independently of which of these quantities is used to set the overall scale- the same results are obtained within a few percent. At finite temperature we use finer lattices down to $\mathrm{a} \leqslant 0.1 \mathrm{fm}$. Our new results confirm completely our previous findings. We compare the results with those of the 'hotQCD' collaboration.
\end{abstract}

\section{Introduction}

An important goal of lattice QCD is to determine the properties of the high temperature quark gluon matter. One wants to determine the temperature scale $\left(T_{c}\right)$, where the ordinary, hadronic matter is supposed to undergo a transition to the high temperature phase. Note that the overall scale (given by $T_{c}$ ) appears in all dimensionful quantities e.g. in the equation of state. Thus, an incorrect $T_{c}$ results in incorrect values for other quantities. Since this transition seems to be a continuous one [1], there is no unambiguous temperature, where the transition takes place. In general different observables may have their characteristic points (e.g. peak position, inflection point) at different temperature values. These temperatures are completely well defined and in principle can be calculated with an arbitrary precision.

Current lattice simulations tend to disagree on these characteristic temperature scales.

When we analyze the nature and/or the absolute scale of the $T>0$ QCD transition for the physically relevant case two ingredients are quite important.

First of all, one should use physical quark masses. The nature of the transition depends on the quark mass, for small or large quark masses it is a first order phase transition, whereas for

${ }^{1}$ speaker 

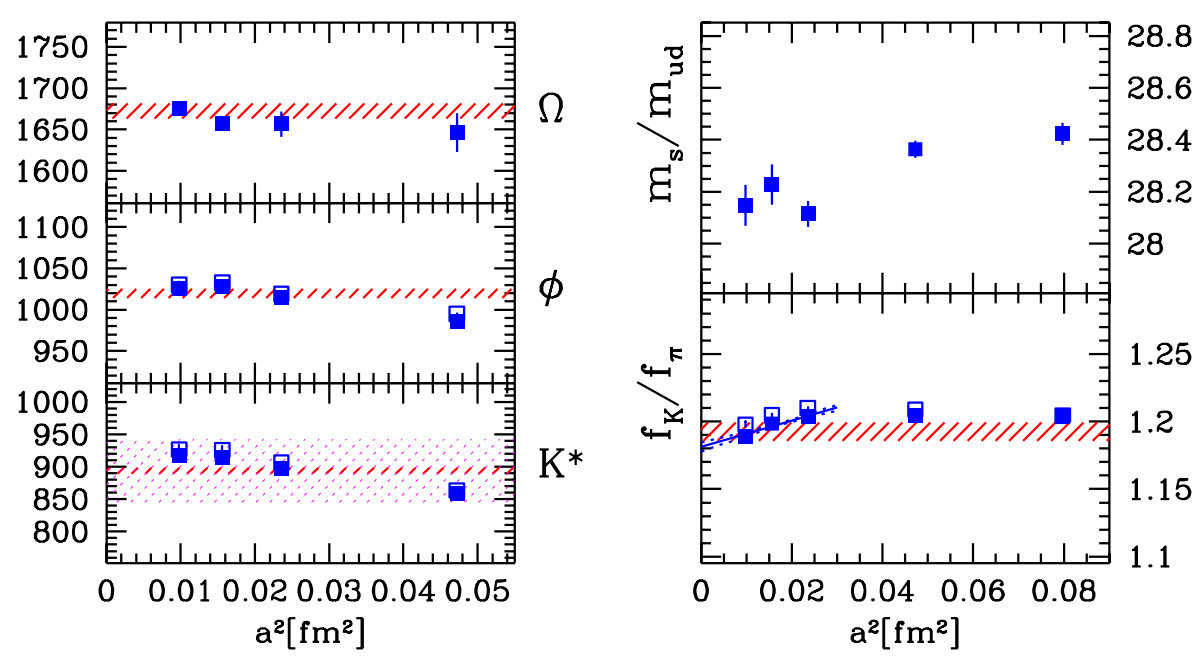

Figure 1: Left panel: masses of $\Omega$ baryon, $\phi(1020)$ meson and $K^{*}(892)$ meson in $\mathrm{MeV}$ on our four finest lattices as a function of the lattice spacing squared. Right panel: quark mass ratio and $f_{K} / f_{\pi}$ for all five ensembles. See text for a detailed explanation.

intermediate quark masses it is an analytic crossover. Since the nature of the transition influences the absolute scale $\left(T_{c}\right)$ of the transition -its value, mass dependence, uniqueness etc. - the use of physical quark masses is also essential for the determination of $T_{c}$, too.

Secondly, the nature of the $T>0$ QCD transition is known to suffer from discretization errors $[2,3]$. The three flavor theory with a large, $a \approx 0.3 \mathrm{fm}$ lattice spacing and standard action predicts a critical pseudoscalar mass of about $300 \mathrm{MeV}$. This point separates the first order and cross-over regions. If we took another discretization, with another discretization error, the critical pseudoscalar mass turns out to be much smaller, well below the physical pion mass of $135 \mathrm{MeV}$. The only way to determine whether the physical point lies in the crossover or firstorder region, and to determine the absolute scale associated with this transition is to carry out a careful continuum limit analysis.

In this paper recent results of the Wuppertal-Budapest group are discussed. Stout-link improved fermionic and Symanzik improved gauge fields are used (for details see Ref. [4]). $T_{c}$ was determined using physical quark masses and a continuum extrapolation. The findings are compared to those of the 'HotQCD' collaboration.

\section{The transition temperature}

An analytic cross-over, like the QCD transition has no unique $T_{c}$, different observables and/or definitions may lead to different temperatures.

In ref. [5] we considered three quantities to locate the transition point: the chiral susceptibility, the strange quark number susceptibility and the Polyakov-loop. The obtained temperatures were 151(3)(3) MeV, 175(2)(4) MeV and 176(3)(4) MeV, respectively. The first errors come from the finite temperature analysis, the second ones from the $T=0$ scale setting. We used physical quark masses and four lattice spacings corresponding to $N_{t}=4,6,8$ and 10. These 

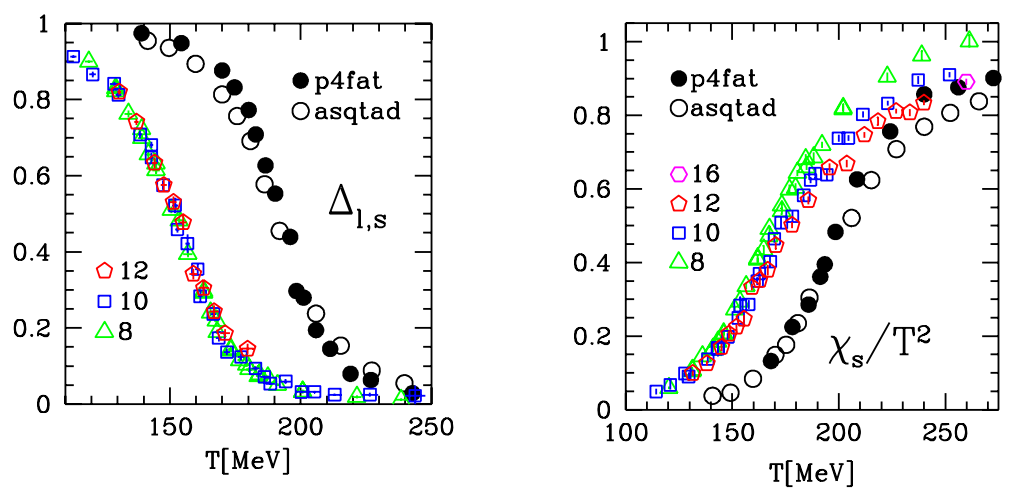

Figure 2: Left panel: renormalized chiral condensate as a function of the temperature. For comparison the 'HotQCD' results are also shown (see text). Right panel: strange quark number susceptibility.

results, especially the one coming from the chiral susceptibilty, are in contradiction with those of ref. [6]. They obtain $T_{c}=192(7)(4) \mathrm{MeV}$ from both the chiral susceptibility and Polyakov loop susceptibility. They used $N_{t}=4$ and 6 lattices with a p4 improved action.

Since then both collaborations improved on their data. We have used physical quark masses also for the $T=0$ simulations and took even finer lattice spacings $\left(N_{t}=12\right.$ and $N_{t}=16$ at one point) at finite emperature [7]. The HotQCD collaboration uses two lattice actions (asqtad and p4) and they extended their simulations to $N_{t}=8$ lattices [8]. In the following we compare the latest results of the two groups to each other.

A possible reason for the discrepancy could be the uncertainty coming from the scale setting. Different quantities can be used to set the lattice spacing and the results should not depend on this choice. Figure 1 shows the masses of the $\Omega$ baryon, $\phi(1020)$ meson and $K^{*}(892)$ meson as well as the ratio of the quark masses and $f_{K} / f_{\pi}$ obtained from our $T=0$ simulations [7]. The agreement to the experimental values shows that using any of the hadron masses to set the lattice spacing would give the same results. The taste symmetry violation (which is an artefact of the staggered lattice formulation and its magnitude reflects the deviation from continuum physics) is quite smaller in [7] than in [8]. As a result the average masses of hadrons are expected to be higher than physical in [8]. This might explain an artificial shift of $T_{c}$ to larger $T$ values in [8].

The light quark chiral condensate $(\langle\bar{\psi} \psi\rangle)$ is minus one times the first derivative of the free energy density with respect to the light quark mass. It is ultraviolet divergent, a possible way of removing divergences was proposed in [9]. If one assumes that the additive divergences of the free energy density depend on the quark masses only through the combination $m_{u d}^{2}+m_{s}^{2}$, then one can get rid of the additive divergences in $\langle\bar{\psi} \psi\rangle$ by using the strange quark condensate $(\langle\bar{s} s\rangle)$. The remaining multiplicative divergences can be removed by dividing with the same quantity at zero temperature

$$
\Delta_{l, s}=\langle\bar{\psi} \psi\rangle-\frac{2 m_{u d}}{m_{s}}\langle\bar{s} s\rangle . \quad \Delta_{l, s} \rightarrow \frac{\Delta_{l, s}(T)}{\Delta_{l, s}(T=0)} .
$$

On Figure 2(left) we plot this quantity as a function of the temperature. There is no significant lattice spacing or volume dependence for lattices of $N_{t}=8,10$ and 12 and for aspect ratios 3-4. For comparison we take the $N_{t}=8$ data of the 'hotQCD' collaboration from [10]. We can see a 


\begin{tabular}{|c|c|c|c|c|c|c|}
\hline & $\Delta \chi_{\bar{\psi} \psi} / T^{4}$ & $\Delta \chi_{\bar{\psi} \psi} / T^{2}$ & $\Delta \chi_{\bar{\psi} \psi}$ & $\Delta_{l, s}$ & $\mathrm{~L}$ & $\chi_{s}$ \\
\hline W'tal-Budapest'09 & $146(2)(3)$ & $152(3)(3)$ & $157(3)(3)$ & $155(2)(3)$ & $170(4)(3)$ & $169(3)(3)$ \\
W'tal-Budapest'06 & $151(3)(3)$ & - & - & - & $176(3)(4)$ & $175(2)(4)$ \\
RBCBC (ref. [6]) & - & $192(4)(7)$ & - & - & $192(4)(7)$ & - \\
\hline
\end{tabular}

Table 1: Continuum extrapolated transition temperatures at the physical point for different observables and in different works. The first three columns give $T_{c}$ obtained from the chiral susceptibility using different normalizations. The other three columns give $T_{c}$ from the renormalized chiral condensate, renormalized Polyakov-loop and the strange quark number susceptibility.

huge disagreement between the curves in the transition regime. The shift between the curves of the different groups is in the order of $35 \mathrm{MeV}$.

The strange quark number susceptibility $\left(\chi_{s}\right)$ is defined as minus one times the derivative of the free energy density with respect to the square of the strange quark chemical potential. It is conveniently normalized by $T^{2}$, by which it will asymptotically reach one as the temperature is increased to infinity (Stefan-Boltzmann limit).

Our results on $N_{t}=8,10$ and 12 are shown in Figure 2(right). We also have an additional point on a very fine lattice $\left(N_{t}=16\right)$ at a high temperature. The comparison with the results of the 'hotQCD' collaboration (see Reference [11]) brings us to a similar conclusion as for the chiral condensate. Around the transition point there is an approximately $20 \mathrm{MeV}$ shift between the results of the two groups. For larger than $\sim 230 \mathrm{MeV}$ temperatures our finer lattices are in good agreement with the 'hotQCD' results.

\section{Conclusions}

Recent results of the Wuppertal-Budapest group were reviewed. Due to the crossover nature of the transition [1] different definitions may lead to different $T_{c}$ values. $T_{c}$ values published by various groups are given in Table 1. Our latest results are consistent with the ones from 2006. The small difference comes from the change in the experimental value of $f_{K}$ since then. The discrepancy with the 'HotQCD' results is still present and has to be resolved by future works.

\section{Acknowledgments}

Computations were performed on the BlueGene at FZ Jülich and on GPU-clusters at Wuppertal and Budapest. This work is supported in part by Hungarian OTKA grant AT049652, DFG grants SFB-TR 55, FO 502/1-2, EU grant (FP7/2007-2013)/ERC n ${ }^{\circ} 208740$.

\section{References}

[1] Y. Aoki, G. Endrodi, Z. Fodor, S. D. Katz, and K. K. Szabo, Nature 443 (2006) 675-678.

[2] P. de Forcrand, S. Kim and O. Philipsen, PoS LAT2007, 178 (2007).

[3] G. Endrodi, Z. Fodor, S. D. Katz and K. K. Szabo, PoS LAT2007, 182 (2007).

[4] Y. Aoki, Z. Fodor, S. D. Katz and K. K. Szabo, JHEP 0601, 089 (2006).

[5] Y. Aoki, Z. Fodor, S. D. Katz and K. K. Szabo, Phys. Lett. B 643, 46 (2006).

[6] M. Cheng et. al., Phys. Rev. D74 (2006) 054507.

[7] Y. Aoki, S. Borsanyi, S. Durr, Z. Fodor, S. D. Katz, S. Krieg and K. K. Szabo, JHEP 0906, 088 (2009).

[8] A. Bazavov et al., arXiv:0903.4379 [hep-lat].

[9] M. Cheng et al., Phys. Rev. D 77, 014511 (2008).

[10] F. Karsch [RBC Collaboration and HotQCD Collaboration], J. Phys. G 35, 104096 (2008).

[11] F. Karsch, PoS C POD07, 026 (2007). 